\title{
Improved algorithm based on modulated wideband converter for multiband signal reconstruction
}

\author{
Min Jia ${ }^{*}{ }^{\dagger}$, Yao Shi ${ }^{\dagger}$, Xuemai Gu, Xue Wang and Zhisheng Yin
}

\begin{abstract}
For the compressed sensing of multiband signals, modulated wideband converter (MWC) is used as the sampling system, and the signal is reconstructed by the simultaneous orthogonal matching pursuit algorithm (SOMP) and its derivative algorithms. In order to find matching atoms, we need to obtain the inner product between atoms in sensing matrix and columns in residual matrix. Next, several inner products corresponding to the same atom constitute an inner product vector. By calculating its 2-norm, we can find the maximum value, whose corresponding atom is the matched atom. However, the inner product actually cannot reflect the relevancy between atoms and residual matrix very accurately, which may eventually lead to wrong results for a certain probability. The main idea of this paper is to change the inner product into the correlation coefficient, so that we can measure the relevancy between atoms and the residual better. Simulation results show that the improved algorithms can get higher probability of the signal reconstruction compared with the original algorithms in the condition of high signal-noise ratio (SNR). It also means that less samples were needed to reconstruct signals than traditional algorithms when the number of bands is unchanged. Since calculating correlation coefficient at each iteration will cost a lot of time, we also proposed a simplified algorithm, which can also improve reconstruction probability and reconstruction time is about the same as corresponding traditional algorithms.
\end{abstract}

Keywords: Modulated wideband converter, Compressed sensing, Multiband, Greedy algorithm, Construction

\section{Introduction}

Traditional compressed sensing theory is mainly used to process discrete and finite-dimensional digital signal. However, it is expected to develop a technology which can sample continuous and infinite dimensional analog signal at sub-Nyquist rate, so as to truly break the bandwidth limitation of existing ADC equipment and reconstruct original signal from the samples of baseband signal after low-pass filters and finally ease the pressure of hardware sampling. To address this problem, a variety of solutions have been proposed. Analog to information convertor (AIC) and Xampling for multiband analog signal [1-5] are two relatively mature technologies among them. Xampling uses modulated wideband converter [6] (MWC) to sample, whose results are infinite measurement vectors

\footnotetext{
*Correspondence: jiamin@hit.edu.cn

${ }^{\dagger}$ Equal contributors

Communication Research Center, Harbin Institute of Technology, Yikuang Street, 150080 Harbin, China
}

(IMV), and it cannot directly reconstruct signal using the traditional reconstruction algorithms. To solve this problem, we can reconstruct the original signal by turning IMV problem into multiple measurement vector (MMV) problem using continuous to finite (CTF) modular [7] under the premise of joint sparse [8].

The simultaneous orthogonal matching pursuit (SOMP) $[9,10]$, treated as a derivative algorithm of matching pursuit simultaneous (OMP) algorithm to solve MMV problem, is what used for reconstruction in CTF at present. Based on this method, many novel MMV algorithms have been proposed according to the derivative algorithms of OMP such as regularized orthogonal matching pursuit (ROMP) [11], stagewise orthogonal matching pursuit (StOMP) [12], compressive sampling matching pursuit (CoSaMP) [13], and subspace pursuit (SP) [14]. The main idea of these algorithms is firstly calculating the inner product between atoms in sensing matrix and columns

\section{实}


in residual matrix. Then, several inner products corresponding to the same atom constitute an inner product vector. By calculating its 2-norm. We can find the maximum value whose corresponding atom is the matched atom. In fact, the inner product cannot measure the relevancy between atoms and residual matrix very well and sometimes will eventually lead to errors.

The idea of this paper is to change the inner product into the correlation coefficient, so that we can measure the matching degree between atoms and the residual better. In order to check the performance of the changement, the SOMP algorithm and its derivative algorithms such as MMV-ROMP, MMV-StOMP, MMV-CoSaMP, and MMV-SP, which can solve MMV problems, are chosen for comparison. The proposed algorithms are improved by changing the inner product into correlation coefficient as screening criterion of atoms, then compare the signal reconstruction probability of these methods with original algorithms. The results show that the improved algorithms have better reconstruction probability and there are less samples required.

\section{Signal model of multiband}

Assuming $x(t)$ is a continuous signal in $L_{2}$ space which satisfies the square integration as follows.

$$
\int_{-\infty}^{+\infty}|x(t)|^{2} d t<+\infty
$$

And its Fourier transformation can be expressed as

$$
X(f)=\int_{-\infty}^{+\infty} x(t) e^{-j 2 \pi f t} d t
$$

If $X(f)$ is finite banded and its frequency spectrum is in $[-1 / 2 T, 1 / 2 T]$, then its Nyquist sampling frequency should be $f_{\mathrm{NYQ}}=1 / T$. Besides, if $X(f)$ satisfies the structure shown in Fig. 1, namely, it contains $N$ disjoint sub-frequency bands whose bandwidths are all less than $B$, then $x(t)$ is a multiband signal.

\section{Modulated wideband converter}

The model of MWC for multiband signal is shown as Fig. 2.

Multiband modulated wideband signal converter is shown in Fig. 2. There are $m$ sampling channels, and the mixing function $p_{i}(t)$ is a pseudo-random sequence with $T_{p}$-period. Its value is $\{+1,-1\}$, and it has $M$ pulses in each period whose interval is $T_{s}$. The values of $p_{i}(t)$ in the

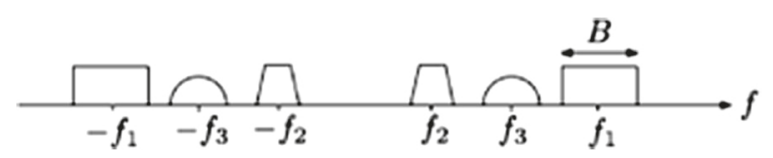

Fig. 1 Model of multiband signal

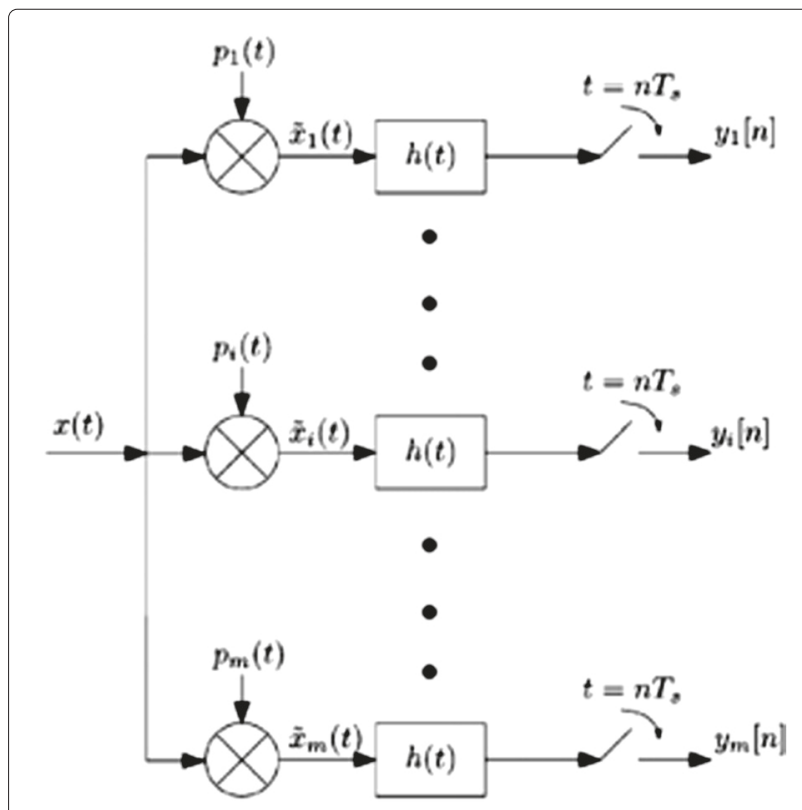

Fig. 2 Model of MWC

first $k$ intervals are denoted as $\alpha_{i k}, h(t)$ is a low-pass filter whose cutoff frequency is $1 / 2 T_{s}$, and the sampling frequency is $1 / T_{s}$. The signal $x(t)$ is transmitted through $m$ channels at the same time, and it is multiplied with different pseudo-random sequences in each branch whose cycle is abiding and obey the same distribution. The samples $y_{i}[n], n=1,2, \ldots, m$ can be obtained after low-pass filters and low-speed sampling.

And the fourier transform can be expressed as

$Y_{i}\left(e^{j 2 \pi f T_{s}}\right)=\sum_{n=-\infty}^{\infty} y_{i}[n] e^{-j 2 \pi f n T_{s}}=\sum_{l=-L_{0}}^{L_{0}} c_{i l} X\left(f-l f_{p}\right)$

where $f_{p}=1 / T_{p}, \mathbf{F}_{s}=\left[-f_{s} / 2, f_{s} / 2\right], L_{0}=\left[\frac{f_{N Y Q}+f_{s}}{2 f_{p}}\right]-1$, and $c_{i l}=\frac{1}{T_{p}} \int_{0}^{T_{p}} p_{i}(t) e^{-j \frac{2 \pi}{T_{p}} l t} d t$.

For convenience, the formula can be expressed in matrix form

$$
\mathbf{y}(f)=\mathbf{A} \alpha(f) f \in \mathbf{F}_{s}
$$

From Eq. (4), it can be seen that $\mathbf{y}(f)$ is a $m \times 1$ dimension vector which is made of $Y_{i}\left(e^{j 2 \pi f T_{s}}\right)$. A is a $M \times L$ dimensional matrix which is composed of $A_{i l}=c_{i,-l}=$ $c_{i l}^{*},-L_{0} \leq l \leq L_{0}, L=2 L_{0}+1$, and $L \approx f_{\mathrm{NYQ}} / B$ in common. Moreover, $\alpha(f)$ is a $L \times 1$ dimensional vector which is composed of $\alpha_{i}(f)=X\left(f+\left(i-L_{0}-1\right) f_{p}\right), f \in \mathbf{F}_{s}$. To recover a signal, we need to firstly solve the sparse spectrum $\alpha(f)$ from Eq. (4). Then, the estimated value $\hat{\mathbf{x}}(t)$ of $\mathbf{x}(t)$ is obtained by using the inverse Fourier transform. However, $f$ is defined over a continuous interval whose 
samples are infinite dimensional vector. Thus, in fact, this is a problem about how to gain the sparse solution from one more of the numerous less demand equations. Moreover, it cannot be calculated by the conventional compressed sensing reconstruction algorithms.Therefore, we should get the support set $S$ of signal by using CTF module and MMV problem can be turned into SMV problem by joint sparsity; then, SOMP algorithm is adopted. In CTF, we first use $\mathbf{y}[n]$ to construct a matrix as follows

$$
\mathbf{Q}=\int_{f \in \mathbf{F}_{s}} \mathbf{y}(f) \mathbf{y}^{H}(f) d f=\sum_{n=-\infty}^{+\infty} \mathbf{y}[n] \mathbf{y}^{T}[n]
$$

where $\mathbf{y}[n]=\left[y_{1}[n], y_{2}[n], \ldots, y_{m}[n]\right]^{T}$, then matrix Q is divided into $\mathbf{Q}=\mathbf{V} \mathbf{V}^{H}$, then we can get framework matrix $\mathbf{V}$. Then, the sparsest support set $\overline{\mathbf{U}}$ of $\mathbf{V}=\mathbf{A U}$ is obtained. According to the support set $\overline{\mathbf{U}}$ and $\mathbf{y}[n]$, we can solve the signal's support set $\mathbf{S}$, and finally, compose the matrix $\mathbf{A s}_{\mathbf{S}}$ by the columns in the matrix $\mathbf{A}$ which correspond to the elements of $\mathbf{S}$, then reconstruct sparse spectrum $\alpha(f)$ by using Eq. (6), so as to reconstruct the traditional signal.

$$
\begin{aligned}
& \alpha_{\mathbf{s}}(f)=\mathbf{A}_{\mathbf{s}}^{\dagger} \mathbf{y}(f) \\
& \alpha_{i}(f)=0, i \notin \mathbf{S}
\end{aligned}
$$

\section{Improved simultaneous orthogonal matching pursuit algorithm}

The reconstruction algorithm for the MWC system is usually simultaneous orthogonal matching pursuit algorithm, whose steps are as follows:

Input: $m \times L$ dimensional sensing matrix $\mathrm{A}$, the number of sub frequency bands $K, m \times 2 K$

dimensional frame vector $\mathbf{V}$, and residual threshold $\theta$. Output: $2 K \times 1$ dimensional support set $\mathbf{S}$.

Initialization: support set $\mathbf{S}=\emptyset$, residual matrix $\mathbf{R}=\mathbf{V}$.

Iteration: Repeat the following steps until the residual is less than the threshold or the number of iteration reach $K$.

(1) $\mathbf{P}=\mathbf{A}^{T} \mathbf{R}$.

(2) $d_{k}=\left\|\mathbf{P}_{k}\right\|_{2}, k=1,2, \ldots, L, \mathbf{P}_{k}$ is $k$ th column of matrix $\mathbf{P}$.

(3) $z_{\mathrm{k}}=\frac{d_{k}}{\left\|\mathbf{A}_{k}\right\|_{2}}, k=1,2, \ldots, L, \mathbf{z}=\left\{z_{1}, z_{2} \ldots, z_{L}\right\}, \mathbf{A}_{k}$ is the $k$ th column of matrix $\mathbf{A}$.

(4) Find the largest item in the vector $\mathbf{z}$ and add its corresponding index $k$ into the support set $\mathbf{S}$, and the symmetric index value $L+1-k$ is also added to the support set $\mathbf{V}$. It is generally considered that the traditional signal is real signal, and its support set is symmetric.

(5) Construct the matrix As corresponding to the columns in matrix $\mathbf{A}$ and support set $\mathbf{S}$.
(6) Evaluate $\hat{\mathbf{U}}, \hat{\mathbf{U}}=\mathbf{A}_{\mathbf{S}}^{\dagger} \mathbf{V}=\left(\mathbf{A}_{\mathbf{S}}^{T} \mathbf{A}_{\mathbf{S}}\right)^{-1} \mathbf{A}_{\mathbf{S}}^{T} \mathbf{V}$.

(7) Update signal residuals $\mathbf{R}=\mathbf{V}-\mathbf{A}_{\mathbf{S}} \hat{\mathbf{U}}$.

It can be found that the inner product between atoms in sensing matrix and columns in residual matrix can be obtained from steps (1) to (4) firstly. Then, several inner products corresponding to the same atom constitute an inner product vector. By calculating its 2-norm, we can find the maximum value, whose corresponding atom is matched atom. The relevancy between residual matrix and sensing matrix actually depends on the relevancy between residual matrix columns and atoms in the sensing matrix. However, because of the randomness of sensing matrix and sampling, the inner product cannot accurately reflect the relevancy between vectors. Wrong atoms can be selected when the number of sampling points is relatively small. However, if the correlation coefficient is used as the criterion, it can reflect the correlation between the random variables better. The reason for the difference can be traced to the calculation method of the inner product and correlation coefficient. For an n dimensional vector $\vec{a}$ and $\vec{b}$, their inner product formula is

$$
\vec{a} \bullet \vec{b}=\sum_{i=1}^{n} a_{i} b_{i}
$$

In two dimensions, the inner product can be expressed as

$$
\vec{a} \bullet \vec{b}=|\vec{a}||\vec{b}| \cos \theta
$$

The inner product itself cannot reflect the relevancy between vectors because it is influenced by the norm of vectors. But if $\vec{a}$ and $\vec{b}$ are the unit vectors, the inner product can reflect the relevancy between $\vec{a}$ and $\vec{b}$. If $\vec{b}$ is regarded as the column vector of the residual matrix, while the vector $\vec{a}$ is regarded as the atom in the matrix $\mathbf{A}$, then, the inner product can reflect the relevancy between the residuals and the atoms.With this relative, the correlation coefficient can also reflect the relevancy, and its formula is

$$
r_{X Y}=\frac{\sum_{i=1}^{N}\left(X_{i}-\bar{X}\right)\left(Y_{i}-\bar{Y}\right)}{\sqrt{\sum_{i=1}^{N}\left(X_{i}-\bar{X}\right)^{2}} \sqrt{\sum_{i=1}^{N}\left(Y_{i}-\bar{Y}\right)^{2}}}
$$

It also excludes the effect of the norm of atoms, and the mean value of atom is zero. Although both the inner product and the correlation coefficient can reflect the relevancy, but in some cases, for the same signal and sensing matrix, the atoms they selected are different, just as the following example: There are two atoms $X=(1,2,3)$, $Y=(2,2,3)$, signal $Z=(4,5,6)$, after normalizing them, we can get $X *, Y *$, and $Z *$. If we use the inner product as screening criterion of atoms, the inner product of $X *$ 
and $Z *$ is 0.975 and the inner product of $Y *$ and $Z *$ is 0.995 .The most matched atoms should be $Y *$. However, when the correlation coefficient is used as screening criterion of atoms, the correlation coefficient of $X *$ and $Z *$ is 1 and the correlation coefficient of $Y *$ and $Z *$ is 0.866 . The most matched atoms should be $X *$. The results are different using two criterions. Then which one is better? To solve this problem, we need to analyze the process of signal reconstruction.

In CTF, the signal support set $\mathbf{S}$ is calculated indirectly by calculating the most sparse solution $\overline{\mathbf{U}}$ of $\mathbf{V}=\mathbf{A U}$, since the support set of $\overline{\mathbf{U}}$ and the support set of signal $\mathbf{y}[n]$ are the same. Then, construct the matrix As corresponding to the columns of matrix $\mathbf{A}$ and support set S. Finally, reconstruct the sparse spectrum $\alpha(f)$ and the original signal by using Eq. (6). If

$$
\mathbf{A}=\left[\begin{array}{ccccccc}
a_{11} & \cdots & a_{1 q} & \cdots & a_{1 i} & \cdots & a_{1 L} \\
0 \vdots & & \vdots & & \vdots & & \vdots \\
a_{r 1} & \cdots & a_{r q} & \cdots & a_{r i} & \cdots & a_{r L} \\
\vdots & & \vdots & & \vdots & & \vdots \\
a_{m 1} & \cdots & a_{m q} & \cdots & a_{m i} & \cdots & a_{m L}
\end{array}\right]
$$

In order to simplify the analysis, $\mathbf{V}=\mathbf{A U}$ is expressed as

$$
\begin{aligned}
& {\left[\begin{array}{ccccccc}
a_{11} & \cdots & a_{1 q} & \cdots & a_{1 i} & \cdots & a_{1 L} \\
\vdots & & \vdots & & \vdots & & \vdots \\
a_{r 1} & \cdots & a_{r q} & \cdots & a_{r i} & \cdots & a_{r L} \\
\vdots & & \vdots & & \vdots & & \vdots \\
a_{m 1} & \cdots & a_{m q} & \cdots & a_{m i} & \cdots & a_{m L}
\end{array}\right] \times} \\
& {\left[\begin{array}{ccccc}
0 & \cdots & 0 & \cdots & 0 \\
\vdots & & \vdots & & \vdots \\
u_{q 1} & \cdots & u_{q t} & \cdots & u_{q p} \\
\vdots & & \vdots & & \vdots \\
u_{i 1} & \cdots & u_{i t} & \cdots & u_{i p} \\
\vdots & & \vdots & & \vdots \\
0 & \cdots & 0 & \cdots & 0
\end{array}\right]=\left[\begin{array}{c}
a_{1 q} u_{q 1}+a_{1 i} u_{i 1} \\
\vdots \\
a_{r q} u_{q 1}+a_{r i} u_{i 1} \\
\vdots \\
a_{m q} u_{q 1}+a_{m i} u_{i 1}
\end{array}\right.} \\
& \cdots a_{1 q} u_{q t}+a_{1 i} u_{i t} \cdots a_{i q} u_{q p}+a_{1 i} u_{i p}
\end{aligned}
$$

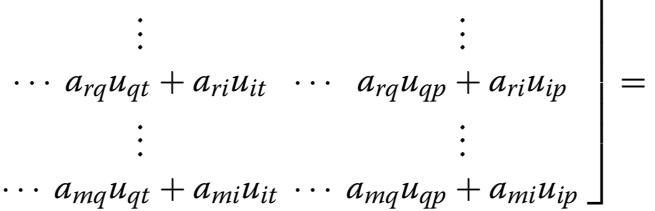

$$
\begin{aligned}
& {\left[A_{q} u_{q 1}+A_{i} u_{i 1} \cdots A_{q} u_{q t}+A_{i} u_{i t} \cdots A_{q} u_{q p}+A_{i} u_{i p}\right]}
\end{aligned}
$$

In which, $A_{q}$ and $A_{i}$ are the $q$ th column and $i$ th column of matrix A, respectively. According to SOMP algorithm, the method of using inner product as screening criterion of atoms can be expressed as

$$
\begin{aligned}
& \lambda=\left\|A_{s} \mathbf{R}\right\|_{2}=\left\|A_{s} \mathbf{V}\right\|_{2}= \\
& \left(\begin{array}{l}
\left(A_{s}^{T} A_{q} u_{q 1}+A_{s}^{T} A_{i} u_{i 1}\right)^{2}+\cdots \\
+\left(A_{s}^{T} A_{q} u_{q p}+A_{s}^{T} A_{i} u_{i p}\right)^{2}
\end{array}\right)^{1 / 2}
\end{aligned}
$$

We hope that when $s=q$ or $s=i, \lambda$ has the maximum value. Since the column vectors of matrix $\mathbf{A}$ are approximately orthogonal, one of $A_{s}^{T} A_{q} u_{q t}$ and $A_{s}^{T} A_{i} u_{i t}$ is relatively large, while the other one is quite small. Assuming

$$
\begin{aligned}
\lambda & \approx \sqrt{\left(A_{s}^{T} A_{q} u_{q 1}\right)^{2}+\cdots+\left(A_{s}^{T} A_{q} u_{q p}\right)^{2}} \\
& =\sqrt{\left(A_{s}^{T} A_{q}\right)^{2}\left(u_{q 1}^{2}+u_{q 2}^{2}+\cdots u_{q p}^{2}\right)}
\end{aligned}
$$

where the value of $\lambda$ is independent of the matrix $\mathbf{U}$, only related to $A_{s}$. Most of the time,

$$
\left(A_{q}^{T} A_{q}\right)^{2}>\left(A_{s}^{T} A_{q}\right)^{2}, s \neq q
$$

But sometimes, the result is opposite (even if the atoms are normalized). Then, the SOMP algorithm will select the wrong atoms. And if the correlation coefficient is used as screening criterion of atoms,

$$
r_{A_{s} A_{q}} \leq 1
$$

if and only if when $s=q$, the equality holds. Thus, using correlation coefficient as screening criterion of atoms is more accurate.

Since the sensing matrix $\mathbf{A}$ and the residual matrix $\mathbf{R}$ are multidimensional, we cannot calculate the correlation coefficient between them directly, so we need to calculate the correlation coefficient between atoms in matrix $\mathbf{A}$ and columns in residual matrix $\mathbf{R}$, and then squaring the correlation coefficients and sum the correlation coefficients corresponding to the same atom; finally, extract the root of the sum and find the largest one, whose corresponding atom is matched atom. This improvement will only change the first step of the SOMP algorithm into $P_{k}=\sum_{i=1}^{m} r_{\mathbf{A}_{k} \mathbf{R}_{i}}$, other steps remain unchanged.

\section{Simulation results}

To test whether the improved algorithm can improve the reconstruction probability, we compare the performance of MMV-ROMP, MMV-StOMP, MMV-CoSaMP, MMV-SP, and SOMP and their improved algorithms. We perform 1000 Monte-Carlo simulation for each algorithm. The signal expression is

$$
x(t)=\sum_{n=1}^{N / 2} \sqrt{B E_{i}} \operatorname{sinc}\left(B\left(t-\tau_{i}\right) \cos \left(2 \pi f_{i}\left(t-\tau_{i}\right)\right)\right.
$$

The signal contains $\mathbf{N}$ frequency bands which are origin symmetric. The maximum bandwidth of sub-frequency 


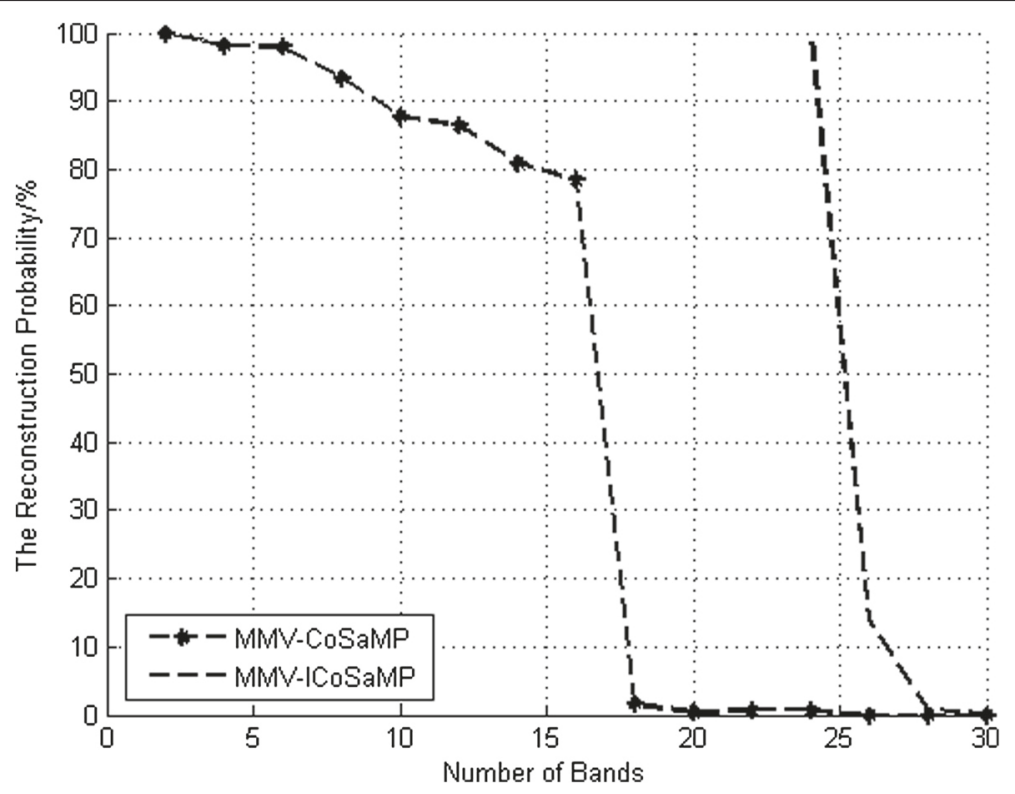

Fig. 3 Reconstruction probability comparison between MMV-CoSaMP and improved MMV-CoSaMP

bands is $50 \mathrm{MHz}$. The carrier frequency $f_{i}$ is a uniform distribution random number among $[-5 \mathrm{GHz}, 5 \mathrm{GHz}]$. The Nyquist sampling rate is $f_{\mathrm{NYQ}}=10 \mathrm{GHz}$. The energy $E_{i}$ of each frequency band is random, time shift $\tau_{i}$ is a uniform distribution random number among $[0,1] \mu s . L_{0}=97$, $L=195, M=195$, SNR $=0$, the sampling period, and the period of the pseudo random sequence $p_{i}(t)$ are $f_{p}=f_{s}=f_{\mathrm{NYQ}} / L=51.28 \mathrm{MHz}$, respectively. The value of $p_{i}(t)$ is either 1 or -1 with equal probability and obey
Bernoulli distribution. The numbers of sampling channels in Figs. 3 and 7 are 100, 100, 50, 50, and 120. The simulation results are as follows.

As can be seen from the simulation, new algorithms are improved in reconstruction probability compared with the traditional algorithms, especially for MMV-ICoSaMP algorithm and MMV-ISP algorithm, the improvement is very significant. When traditional algorithms are completely unable to reconstruct the signal, as is shown in

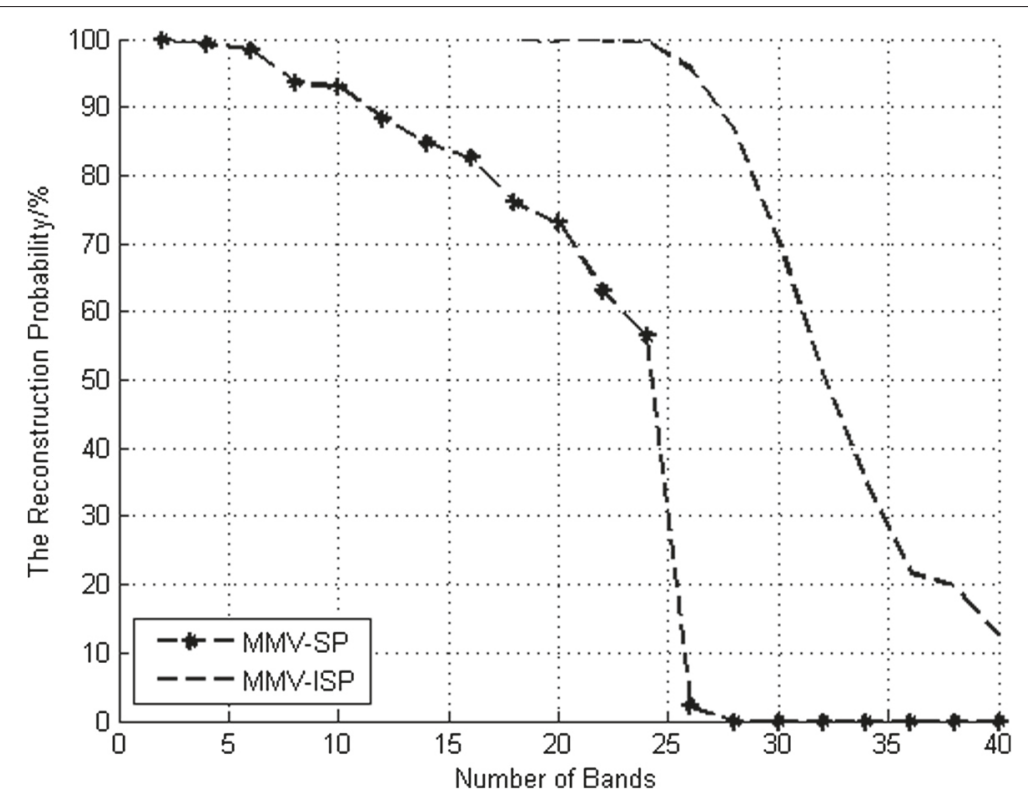

Fig. 4 Reconstruction probability comparison between MMV-SP and improved MMV-SP 


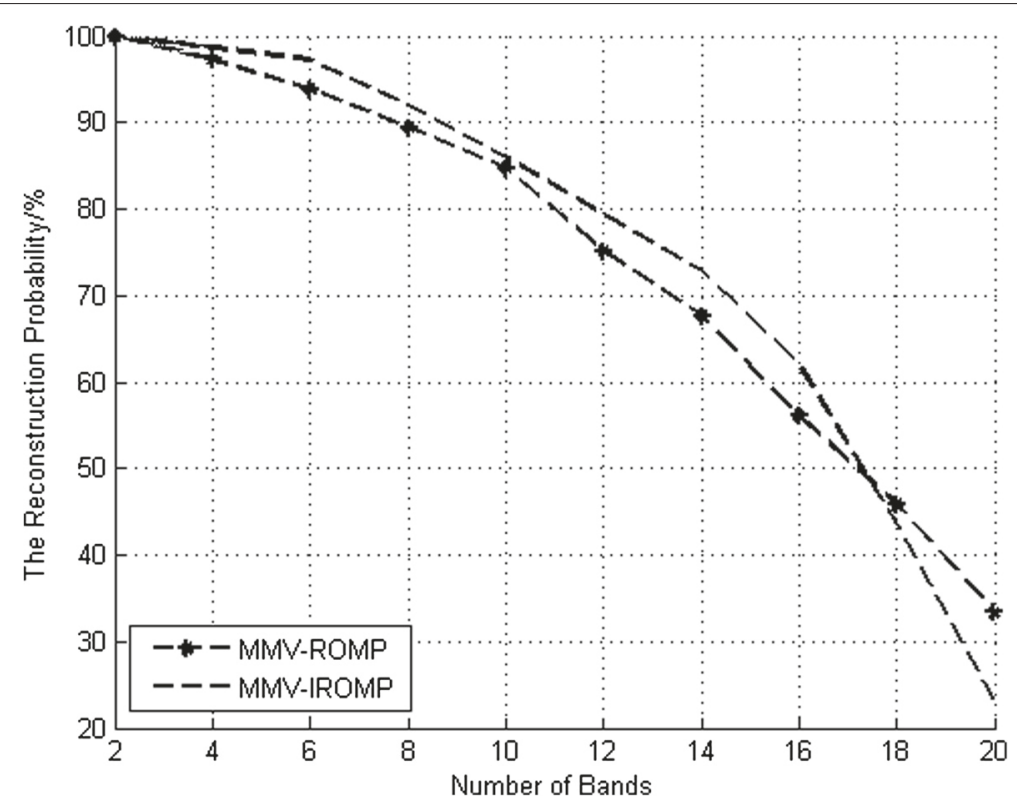

Fig. 5 Reconstruction probability comparison between MMV-ROMP and improved MMV-ROMP

Figs. 3 and 4, the improved algorithm still can reconstruct the signal with $100 \%$ probability. It also means that less samples were needed to reconstruct signals than traditional algorithms when the number of bands is unchanged. The other three algorithms can also improve the reconstruction probability to a limited extent, as is shown in Figs. 5, 6 and 7. Because of the improvement about correlation coefficient, the complexity of the algorithm is increased. In addition, relatively small SNR has an adverse effect on reconstruction probability of improved algorithm, as is shown in Figs. 8 and 9, where the number of sampling channels is 100 and the number of bands is 20 .

Although changing the inner product into correlation coefficient as screening criterion of atoms can improve the performance of the algorithm, calculating correlation

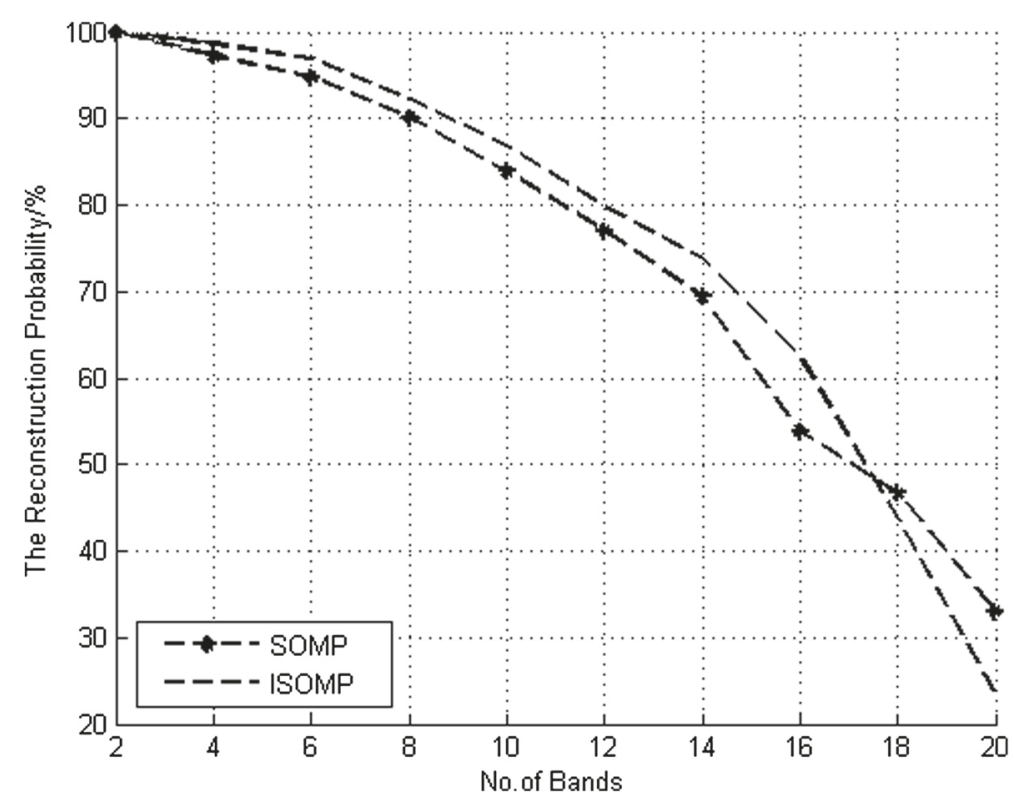

Fig. 6 Reconstruction probability comparison between SOMP and improved SOMP 


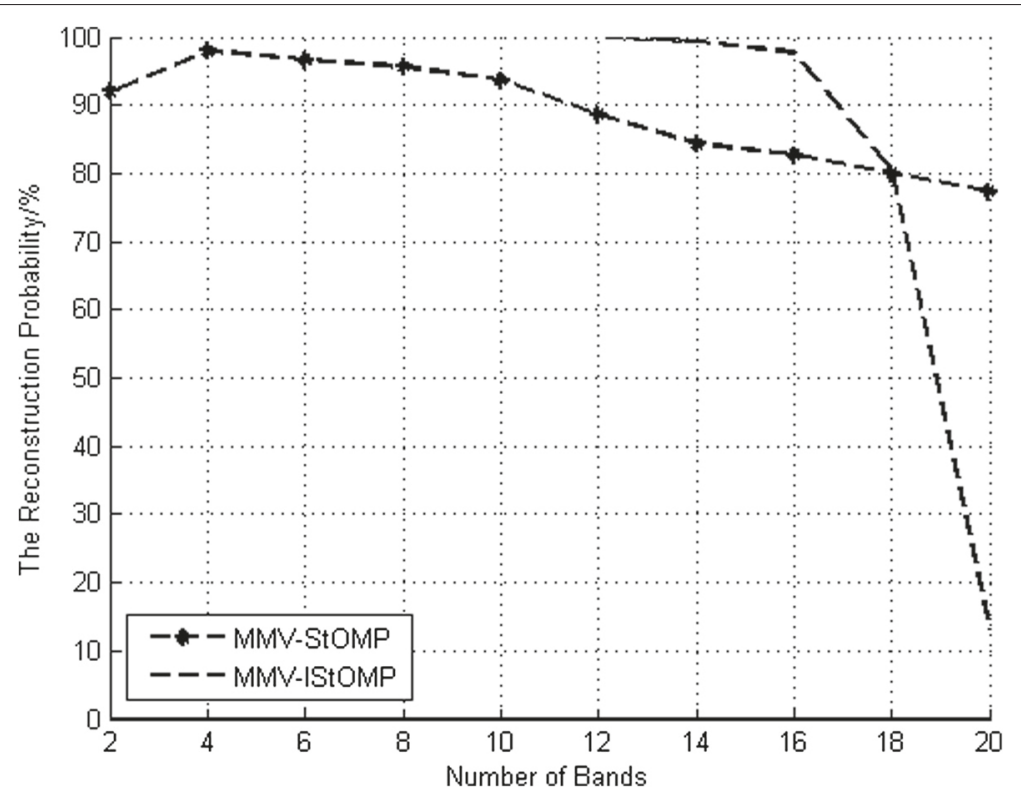

Fig. 7 Reconstruction probability comparison between MMV-StOMP and improved MMV-StOMP

coefficient at each iteration will cost a lot of time, so we can consider the possibility of simplified this process. A significant difference between the inner product and correlation coefficient is that correlation coefficient excludes the impact of data mean, so we can consider whether it is feasible if the column vectors of sensing matrix are centered or normalized firstly and still use inner product as screening criterion of matching atoms. Simulation result shows that compared to traditional MMV-SP algorithms, normalization cannot improve the reconstruction probability, but centralization can. If the column vectors of sensing matrix are centered firstly and still use inner product as screening criterion of matching atoms, the reconstruction probability is silently worse than MMV-ISP algorithm, just as shown in Fig. 10, where MMV-SISP means simplified MMV-ISP. The advantage of this simplification

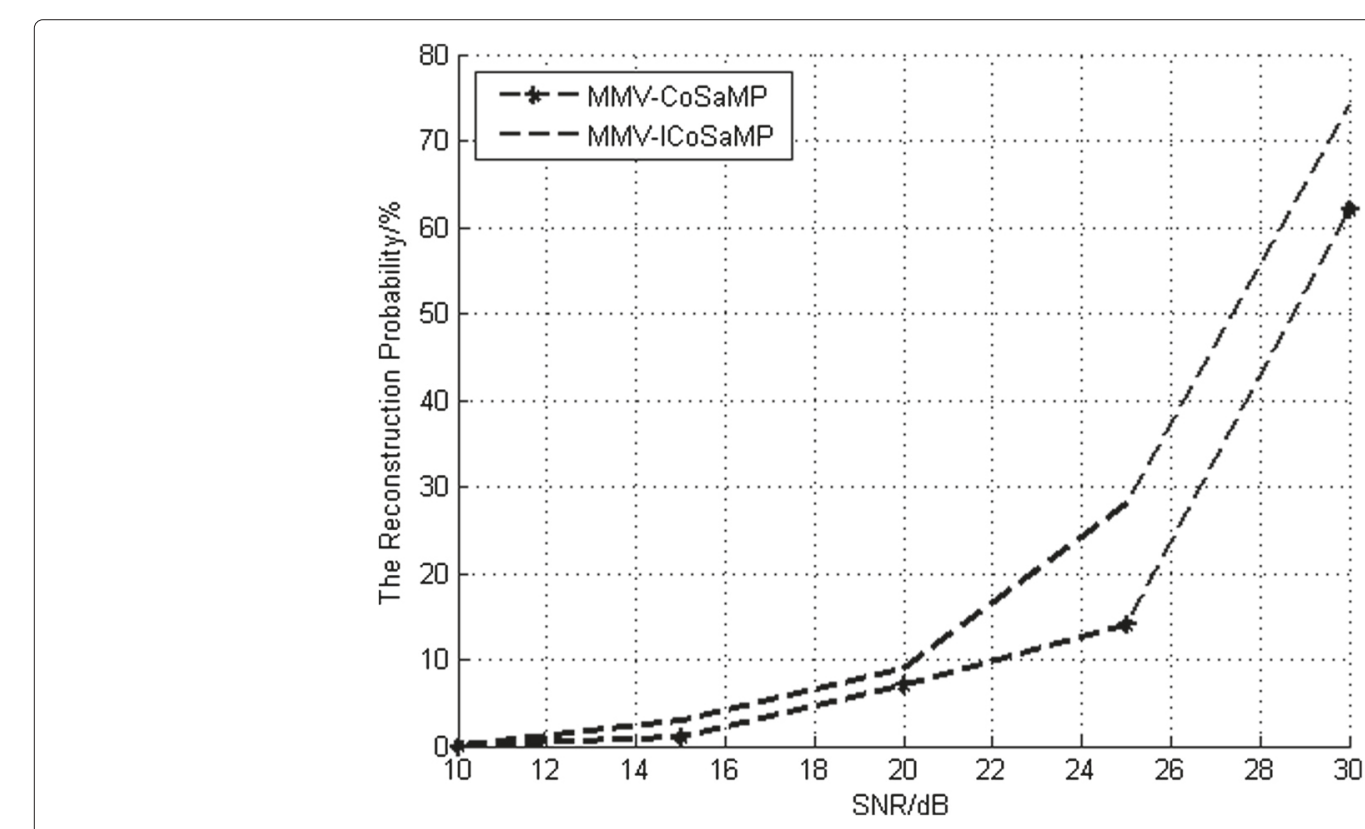

Fig. 8 Reconstruction probability comparison between MMV-CoSaMP and improved MMV-CoSaMP in different SNRs 


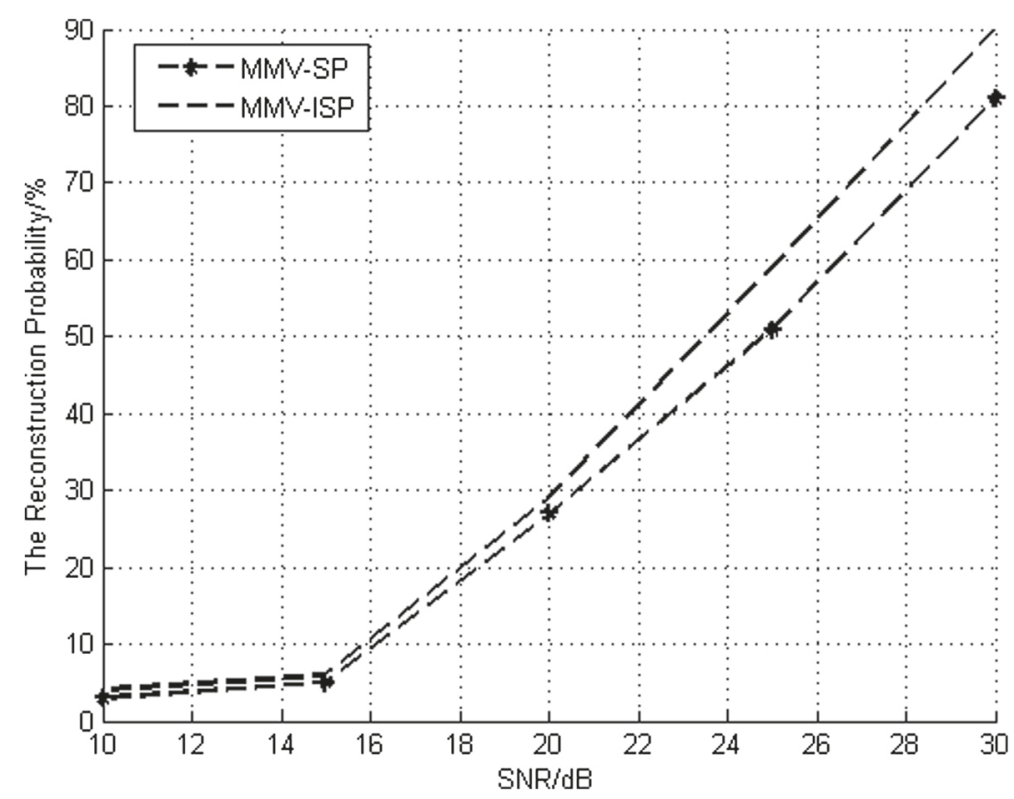

Fig. 9 Reconstruction probability comparison between MMV-SP and improved MMV-SP in different SNRS

is that it can reduce the complexity of the proposed algorithm so that the reconstruction time is about the same as MMV-SP algorithm.

\section{Conclusions}

In order to improve the reconstruction probability of MWC sampling system, we improve the SOMP algorithm and its derivative algorithms in this paper by changing the inner product into correlation coefficient as screening criterion of atoms. The simulation experiments show that the improved algorithm can increase the reconstruction probability, especially for MMV-ICoSaMP algorithm and MMV-ISP algorithm, the improvement is very significant. For SOMP, MMV-ROMP, and MMV-StOMP algorithms, the improvement can also increase the reconstruction probability to a limited extent. It also means that less samples were needed to reconstruct signals than traditional algorithms when the number of bands is unchanged.

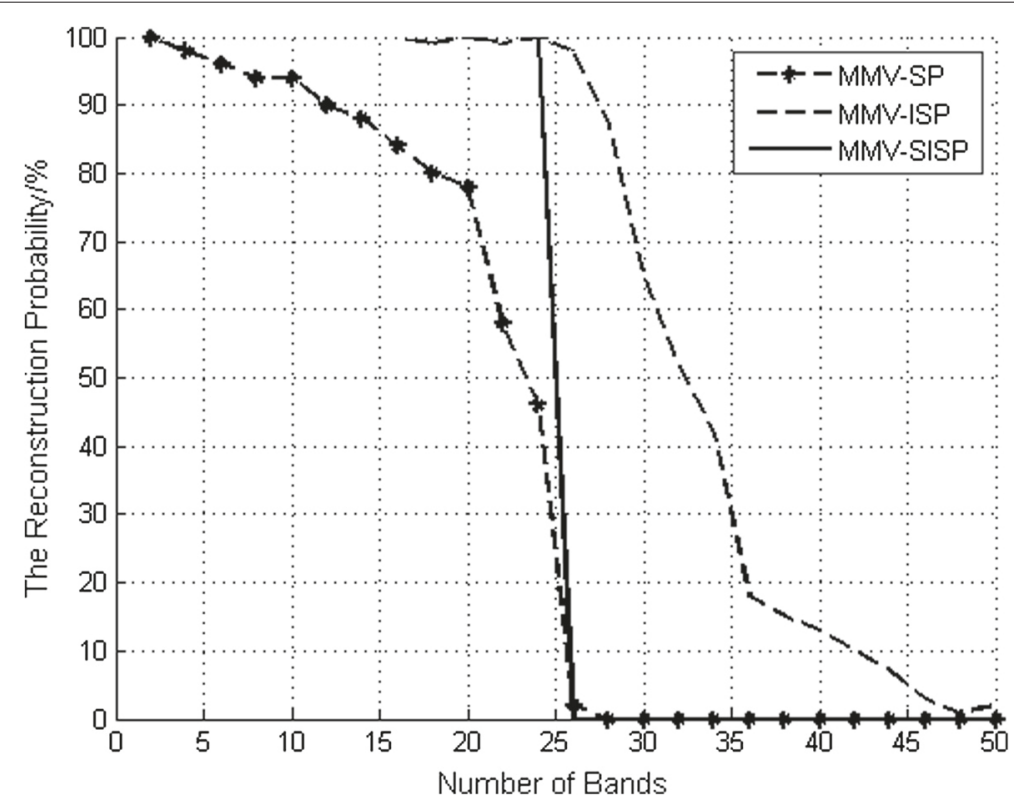

Fig. 10 Comparison of the reconstructed probability of the simplified and unsimplified algorithms 
Because of the improvement of correlation coefficient, the complexity of the algorithms increase, so the reconstruction time correspondingly increase and the algorithm is not suitable for the system with high real-time requirements. For systems that require high accuracy, the improved algorithms can be used. Since calculating correlation coefficient at each iteration will cost a lot of time, we also proposed a simplified algorithm, which can also improve reconstruction probability and reconstruction time is about the same as traditional algorithms. In addition, relatively small SNR has an adverse effect on reconstruction probability of improved algorithms, so the improved algorithms are more suitable for low noise channels.

\section{Competing interests}

The authors declare that they have no competing interests.

\section{Acknowledgements}

This work was supported in part by National Natural Science Foundation of China under Grants No. 61201143 and No. 91438205.

Received: 1 December 2015 Accepted: 4 February 2016

Published online: 16 February 2016

\section{References}

1. M Mishali, YC Eldar, Blind multiband signal reconstruction: compressed sensing for analog signals. IEEE Signal Proc. Mag. 57(3), 993-1009 (2009)

2. M Mishali, YC Eldar, A Elron, Xampling: signal acquisition and processing in union of subspaces. IEEE Transac. Signal Process. 59(10), 4719-4734 (2011)

3. M Mishali, YC Eldar, O Dounaevsky, E Shoshan, Xampling: analog to digital at sub-Nyquist rates. IET J. Circ. Devices and Syst. 5(1), 8-20 (2011)

4. XXiong, K Zheng, R Xu, W Xiang, P Chatzimisios, Low power wide area machine-to-machine networks: key techniques and prototype. IEEE Commun. Mag. 53(9), 64-71 (2015)

5. G Wang, W Xiang, J Yuan, Generalized wireless network coding schemes for multi-hop two-way relay channels. IEEE Transac. Wirel. Commun. 13(9), 5132-5147 (2014)

6. K Gedalyahu, R Tur, YC Eldar, Multichannel sampling of pulse streams at the rate of innovation. IEEE Transac. Signal Process. 59(4), 1491-1504 (2011)

7. M Mishali, YC Eldar, From theory to practice: sub-Nyquist sampling of sparse wideband analog signals. IEEE J. Sel. Topics. Signal Process. 4(2), 375-391 (2010)

8. M Mishali, YC Eldar, Reduce and boost: recovering arbitrary sets of jointly sparse vectors. IEEE Transac. Signal Process. 56(10), 4692-4702 (2008)

9. JA Tropp, AC Gilbert, MJ Strauss, Algorithms for simultaneous sparse approximation. Signal Process. 86(3), 572-588 (2006)

10. JA Tropp, AC Gilbert, MJ Strauss, in Proceedings of the 42th Asilomar Conference on Signals, Systems, and Computers. Simultaneous sparse approximation via greedy pursuit (IEEE, Pacific Grove, California, USA, 2008), pp. 721-724

11. D Needell, R Vershynin, Uniform uncertainty principle and signal recovery via regularized orthogonal matching pursuit. Found. Comput. Math. 9(3), 317-334 (2009)

12. DL Donoho, Y Tsaig, Droril, JL Starck, Sparse solution of underdetermined linear equations by stagewise orthogonal matching pursuit. IEEE Trans. Inf. Theory. 58(2), 1094-1121 (2012)

13. W Dai, O Milenkovic, Subspace pursuit for compressive sensing signal reconstruction. IEEE Trans. Inf. Theory. 55(5), 2230-2249 (2009)

14. D Needell, JA Tropp, CoSaMP: iterative signal recovery from incomplete and inaccurate samples. Appl. Comput. Harmonic Anal. 26, 301-321 (2009)

\section{Submit your manuscript to a SpringerOpen ${ }^{\circ}$ journal and benefit from:}

- Convenient online submission

- Rigorous peer review

- Immediate publication on acceptance

- Open access: articles freely available online

- High visibility within the field

- Retaining the copyright to your article

Submit your next manuscript at springeropen.com 